\title{
Serotonin Transporter and Tryptophan Hydroxylase Gene Variations Mediate Working Memory Deficits of Cocaine Users
}

\author{
Michael M Havranek', Matthias Vonmoos ${ }^{2}$, Christian P Müller ${ }^{3}$, Jessica R Büetiger², Eve Tasiudi ${ }^{4}$, \\ Lea M Hulka ${ }^{2}$, Katrin H Preller ${ }^{2}$, Rainald Mössner ${ }^{5}$, Edna Grünblatt ${ }^{4,6}$, Erich Seifritz ${ }^{1,6}$ and \\ Boris B Quednow*,2,6
}

'Department of Psychiatry, Psychotherapy and Psychosomatics, Psychiatric Hospital of the University of Zurich, Zurich, Switzerland; ${ }^{2}$ Experimental and Clinical Pharmacopsychology, Department of Psychiatry, Psychotherapy and Psychosomatics, Psychiatric Hospital of the University of Zurich, Zurich, Switzerland; ${ }^{3}$ Department of Psychiatry and Psychotherapy, Friedrich-Alexander-University Erlangen-Nuremberg, Erlangen, Germany; ${ }^{4}$ University Clinic for Child and Adolescent Psychiatry, University of Zurich, Zurich, Switzerland; ${ }^{5}$ Department of Psychiatry and Psychotherapy, University of Tuebingen, Tuebingen, Germany; ${ }^{6}$ Neuroscience Center Zurich, University and ETH Zurich, Zurich, Switzerland

\begin{abstract}
Cocaine users consistently develop working memory (WM) impairments but the mediating molecular mechanisms are unknown so far. Recent evidence suggests that the serotonin $(5-\mathrm{HT})$ system is altered by chronic cocaine use, while also being involved in WM processing. Thus, we investigated the effects of genetic variations impacting 5-HT activity and of peripheral 5-HT transporter (5-HTT) mRNA expression on WM performance in cocaine users and stimulant naive controls. Two hundred twenty participants ( 126 cocaine users, 94 controls) were assessed with visuospatial, spatial, and verbal WM tasks, genotyped for the length polymorphism in the promoter region of the 5-HTT (5-HTTLPR), the variable number of tandem repeats in the second intron of the 5-HTT (VNTR In2), two single-nucleotide polymorphisms (rs4570625 and rs 1386497) in the tryptophan hydroxylase-2 (TPH2) gene and quantified for peripheral 5-HTT mRNA expression in whole-blood samples. Several significant gene $\times$ environment interactions between 5-HT genotypes and cocaine use on WM emerged: in cocaine users, the long/long (5-HTTLPR), 9+ 10/9+ 10 (VNTR In2) and C/C (TPH2 rs I 386497) genotypes were risk alleles for WM impairments, whereas in healthy controls these polymorphisms were associated with improved WM performance. Analogously, high 5-HTT mRNA levels were associated with worse executive WM performance in cocaine users but with increased performance in controls. These gene $\times$ environment interactions suggest that the 5-HT system has an important role in the development of cognitive deficits in chronic cocaine users. Hence, pharmacological compounds targeting 5-HT neurotransmission might be promising for the treatment of cognitive deficits in cocaine dependence.
\end{abstract}

Neuropsychopharmacology (2015) 40, 2929-2937; doi:I 0.I038/npp.20I5.I46; published online 24 June 2015

\section{INTRODUCTION}

Cocaine users consistently show impairments in several cognitive domains such as attention, working memory (WM), declarative memory, and executive functions (Goldstein $e t$ al, 2004; Beveridge et al, 2008; Vonmoos et al, 2013). Among these, WM seems to be affected first and to show greatest deficits after ongoing use (Jovanovski et al, 2005; Vonmoos et al, 2013). Thus, WM was claimed to be particularly susceptible to cocaine consumption (Robbins and Arnsten, 2009; Vonmoos et al, 2013). Recent longitudinal data, furthermore, suggest that WM deficits in cocaine users are

*Correspondence: Professor BB Quednow, Experimental and Clinical Pharmacopsychology, Department of Psychiatry, Psychotherapy and Psychosomatics, Psychiatric Hospital of the University of Zurich, Lenggstrasse 31, CH-8032 Zurich, Switzerland, Tel: +4I 443842777 , Fax: +4I 44384 3396, E-mail: quednow@bli.uzh.ch

Received 9 February 20 15; revised 5 May 2015; accepted I 8 May 20 15; accepted article preview online 27 May 2015 likely drug-induced rather than predisposed (Vonmoos et al, 2014). However, the molecular mechanism by which such cognitive deficits develop remains unclear so far.

Increasing evidence from rodent and human studies suggests that the serotonin (5-HT) system has an important role in WM processing (Fitzgerald, 2011). For instance, altering 5-HT synthesis by tryptophan depletion revealed that 5-HT transmission positively correlates with activity in the prefrontal cortex (PFC) and with WM performance (Porter et al, 2003; Allen et al, 2006). These effects are assumed to be mediated by $5-\mathrm{HT}_{2 \mathrm{~A}}$ and $5-\mathrm{HT}_{1 \mathrm{~A}}$ receptors in the PFC (Williams et al, 2002; Puig and Gulledge, 2011). Moreover, in genetic studies, the length polymorphism (short $v s$ long allele) in the promoter region of the serotonin transporter (5-HTT) gene (5-HTTLPR and SLC6A4) and more recently a single-nucleotide polymorphism (SNP, rs4570625) in the promoter region of the tryptophan hydroxylase-2 (TPH2) gene have been found to influence WM performance (Reuter et al, 2008; Bosia et al, 2010; 
Homberg and Lesch, 2011; Zilles et al, 2012). This is plausible because the 5-HTT terminates 5-HT from the synaptic cleft by 5 -HT reuptake after synaptic signaling (Lesch et al, 1996; Glatz et al, 2003) and the TPH2 (the ratelimiting enzyme of 5-HT synthesis) appears to facilitate 5-HT neurotransmission by increasing 5-HT availability (Kim et al, 2002). However, results of the genetic studies are inconsistent. Some authors found the long allele of 5-HTTLPR to be associated with superior WM performance (Bosia et al, 2010; Zilles et al, 2012), whereas others claimed the short allele to be associated with improved performance (Enge et al, 2011; Anderson et al, 2012). It was argued that these conflicting results may either be due to a task specificity of the 5-HTTLPR (Anderson et al, 2012; Zilles et al, 2012) or due to undetected and moderating environmental factors (Zilles et al, 2012).

The 5-HTTLPR has been intensively investigated regarding its potential impact on the risk for cocaine addiction but the results are mixed showing either and association of the long allele with cocaine dependence only in European patients (Patkar et al, 2002) or an association of the short allele with cocaine/heroin dependence in Americans of African descent (Enoch et al, 2011) or no significant associations of the 5-HTTLPR with cocaine addiction and treatment outcome (Mannelli et al, 2005; Patkar et al, 2001; Tristan-Noguero et al, 2013). Also, polymorphisms in the TPH2 gene have been analyzed but they did not show any associations with cocaine dependence (Dahl et al, 2006).

Several rodent studies have shown that 5-HT neurotransmission is drastically increased after acute and reduced after chronic cocaine administration (for a review see: Müller and Homberg, 2015). Positron emission tomography (PET) studies in monkeys showed increased 5-HTT availability in frontal areas after chronic cocaine administration, whereas PET studies in humans revealed that chronic use is associated with reduced $5-\mathrm{HT}_{1 \mathrm{~B}}$ receptor binding in the PFC (Gould et al, 2011; Matuskey et al, 2013). In addition, studies in rats demonstrated that the TPH2 activity in the raphe nucleus increases after chronic cocaine administration (Vrana et al, 1993). In their comprehensive review on the role of 5-HT in drug addiction, Müller and Homberg (2015) concluded that chronic cocaine use might induce an increase in terminal 5-HTT function with reduced extracellular 5-HT levels in cortical areas. Subsequent acute cocaine challenges may then encounter more terminal and less somatodendritic 5-HTT targets and, thus, induce an intensified cortical 5-HT increase further reiterating the change in 5-HT function.

The aim of this study was to investigate whether 5-HTT and TPH 2 polymorphisms are associated with WM deficits in cocaine users. As outlined above, polymorphisms in 5-HTT and TPH2 genes (Reuter et al, 2008; Bosia et al, 2010; Homberg and Lesch, 2011; Zilles et al, 2012) as well as chronic cocaine use (Müller and Homberg, 2015) are assumed to impact 5-HT neurotransmission. Given that the 5-HT system has been linked to WM performance (Porter et al, 2003; Allen et al, 2006), we hypothesized that 5-HTT and TPH2 polymorphisms interact with cocaine use regarding $\mathrm{WM}$ performance. In addition to the abovementioned 5-HTTLPR and the TPH2 polymorphism (rs4570625), we further examined the variable number tandem repeats polymorphism within intron 2 of the
5-HTT (VNTR In2) and a second promising SNP in the TPH2 gene (rs1386497). The VNTR In 2 was chosen because it is assumed to have 5-HTT transcription regulating properties and the rs1386497 was selected due to its association with cognitive deficits in attention deficit hyperactivity disorder (ADHD) (MacKenzie and Quinn, 1999; Manor et al, 2008). Besides these polymorphisms, we measured 5-HTT (SLC6A4) mRNA expression in peripheral blood in order to detect potential interactions between 5-HTT and TPH2 genotype, 5-HTT gene expression and cocaine use. Finally, because of the inconsistent results of previous studies, we used various WM tasks in order to compare genotype effects for visuospatial, spatial, and verbal WM subdomains.

\section{MATERIALS AND METHODS}

\section{Participants}

A total sample of 220 participants (126 chronic cocaine users and 94 stimulant naive controls) were investigated as a part of the longitudinal Zurich Cocaine Cognition Study $\left(\mathrm{ZuCo}^{2} \mathrm{St}\right.$ : (Vonmoos et al, 2013; Vonmoos et al, 2014)). Cocaine users were included if they indicated cocaine as their primary drug of choice, showed a use of $>0.5$ g per month, and a maximum abstinence duration no longer than 6 months. Exclusion criteria for all participants were any current or previous neurological disorders or head injuries and use of prescription drugs affecting cognitive functioning. Additional exclusion criteria for the cocaine users were regular use of opioids or a polytoxic drug use pattern according to DSM-IV as well as any current or previous Axis-I DSM-IV psychiatric disorders with exception of cocaine and alcohol abuse/dependence, a history of depression, and ADHD. Specific exclusion criteria for the control group were regular drug use (lifetime $>15$ occasions) with the exception of nicotine dependence and occasional cannabis use and any current or previous Axis-I DSM-IV psychiatric disorder (American Psychiatric Association, 1994). Participants self-reports regarding drug use were controlled by toxicological analyses of urine and $6 \mathrm{~cm}$ (if possible) hair samples (see also Supplementary Table S1). More detailed information about selection, recruitment, and drug screenings have been presented in detail in our previous work (Preller et al, 2013; Vonmoos et al, 2013; Hulka et al, 2014). The presented study was approved by the Cantonal Ethics Committee of Zurich. All participants provided written informed consent before the study and were financially compensated for their participation.

\section{Procedure}

All participants were examined by trained psychologists using a Structural Clinical Interview (SCID-I; Wittchen et al, 1997) according to DSM-IV. Drug use was assessed using a structured and standardized Interview for Psychotropic Drug Consumption (Quednow et al, 2004). Subsequently, participants underwent a battery of neuropsychological evaluations including the Spatial Working Memory (SWM) task and the Paired Associates Learning (PAL) task from the Cambridge Neuropsychological Test Automated Battery (CANTAB, www.cambridgecognition.com; Strauss et al, 2006) as well as the Letter Number Sequencing Task (LNST) (Wechsler, 1997). In order to draw conclusions about global WM 
processes, the three main parameters of these tests (SWM total errors, PAL first trial memory score, and LNST correct responses) were z-transformed (based on means and S.D. of the control group) and incorporated into a global WM capacity index (as described in our previous work: Vonmoos et al, 2013). To additionally investigate an executive WM component, we separately analyzed the SMW strategy score that is thought to primarily reflect executive functioning, ie, strategic planning (Owen et al, 1990).

\section{Genotyping and mRNA Quantification}

The 5-HTTLPR, VNTR In2, and TPH2 polymorphisms were determined as described in the Supplementary Material. The 5-HTTLPR was assessed in its biallelic (short vs long allele) instead of its triallelic form (LA vs LG, SA, and SG, see: Hu et al, 2006) in order to compare our results with previous studies on the effects of 5-HTTLPR on WM. Furthermore, subjects with nine copies $(2.7 \%)$ in the VNTR In2 were pooled with subjects carrying 10 copies resulting in three genotype groups: $9+10 / 9+10$ vs $9+10 / 12$ vs 12/12. Also, subjects with the rare $\mathrm{T} / \mathrm{T}$ genotype $(6,2 \%)$ of $\mathrm{TPH} 2$ rs4570625 and the rare $\mathrm{C} / \mathrm{C}$ genotype (3.6\%) in TPH2 rs1386497 were pooled with subject with the G/T and the $\mathrm{A} / \mathrm{C}$ genotype, respectively, resulting in the two genotype groups: $\mathrm{G} / \mathrm{G}$ vs $\mathrm{G}+\mathrm{T} / \mathrm{T}$ and $\mathrm{A} / \mathrm{A}$ vs $\mathrm{A}+\mathrm{C} / \mathrm{C}$.

Total RNA was isolated from whole blood using the RNA isolation NucleoSpin RNA Blood in combination with the NucleoSpin RNA/DNA Buffer Set according to the manufacturer's recommendation (Macherey-Nagel AG, Oersingen, Switzerland). Total RNA samples were spectrophotometrically scanned (NanoVue, GE Healthcare Life Sciences) to obtain the A260/A280 of >1.9 and concentration levels. In addition, for RNA quality resulting with RNA integrity number values, all samples were measured on the automated electrophoresis system (Experion, BioRad Co., Hercules, CA). Quantitative real-time RT-PCR was conducted for 5-HTT (SLC6A4) and six additional reference genes (ACTB, GAPDH, ALAS1, RPL13A, PPIA, and $18 \mathrm{~S}$ ribosomal RNA) as described previously (Grunblatt et al, 2009). Total RNA (500 ng) from each sample was reverse transcribed using iScript cDNA synthesis kit (BioRad Co.). Each amplification was performed in a total volume of $20 \mu \mathrm{l}$ containing $5 \mu \mathrm{l}$ QuantiTect SYBR Green PCR kit (Qiagen) and the specific primer mix (PrimerAssay-Qiagen). PCR conditions were run according to the manufacturer's manual (Qiagen). A melting point analysis was conducted for each assay to confirm specificity of PCR products and all PCR reactions were run in triplicates. The program LinRegPCR (www.hartfaalcentrum.nl) was used to determine the PCR efficiency. Gene expression and normalization analysis with the most stable reference genes were conducted using the QBase plus software (Biogazelle; Vandesompele et al, 2002). The software detected that the reference gene RPL13A was least stable and, therefore, this gene was excluded and normalization analysis was conducted using the five other reference genes. We also tried to determine TPH2 mRNA but the melting point analysis revealed an insufficient quality of the PCR products indicating that the TPH2 mRNA was degraded.

\section{Statistical Analyses}

Global and task-specific WM effects were analyzed separately for each polymorphism using analyses of variance (ANOVA) with the factors: group (cocaine users vs controls) and genotype (5-HTTLPR and VNTR In2: threefold, TPH2 rs4570625 and rs1386497: twofold, see above). In addition, analyses of covariance (ANCOVA) with the covariates age, years of education, $\mathrm{BDI}$, and $\mathrm{ADHD}$ were calculated to investigate possible influences of these factors. The influence of 5-HTT mRNA expression on WM performance was investigated using Pearson correlations, whereas mRNA expression $\times$ genotype interactions were examined using ANCOVAs with mRNA expression as the covariate. Results were considered significant if $p<0.05$ after correction for multiple comparisons using the Hochberg method (Hochberg, 1988). Hochberg's procedure (a step-up modification of the Bonferroni method) tests each partition hypothesis using all the order statistics by formulating a sequence of critical values based on Simes' inequality. Statistical analyses were conducted with SPSS (Version 20.0), testing of linkage disequilibrium (LD) between polymorphisms was performed with the software Haploview (Barrett et al, 2005) and associations between polymorphisms and cocaine use was analyzed with the Armitage's Trend Test.

\section{RESULTS}

\section{Demography and Association Analysis}

Cocaine users and stimulant naive controls did not differ regarding age and sex distribution. However, cocaine users had significantly fewer years of education, higher scores in the Beck Depression Inventory (BDI), and higher scores of ADHD than controls (see Table 1). Beyond these group differences, we did not find any significant genotype or group $\times$ genotype interaction effects on BDI (all $p>0.05)$ or

Table I Demographic Data of Healthy Controls and Cocaine Users (Means and SD)

\begin{tabular}{|c|c|c|c|c|c|}
\hline Demographic data & Controls $(n=94)$ & Cocaine users $(n=126)$ & t-test $/ \chi^{2}$ & df & $p$ \\
\hline Age, mean (SD) & $30.0(8.8)$ & $30.1(8.9)$ & -0.1 & 218 & 0.917 \\
\hline Sex (male/female) & $68 / 26$ & $91 / 35$ & 0.0 & । & 0.554 \\
\hline Years of education, mean (SD) & $10.7(1.8)$ & $10.2(1.7)$ & 2.1 & 218 & 0.041 \\
\hline BDI Score, mean (SD) & $4.2(4.0)$ & $8.6(7.1)$ & -5.8 & 204.2 & 0.000 \\
\hline ADHD DSM-IV Score, mean (SD) & $7.9(5.0)$ & $14.4(9.4)$ & -6.5 & 198.7 & 0.000 \\
\hline
\end{tabular}

Abbreviations: ADHD, attention deficit hyperactivity disorder; BDI, Beck Depression Inventory.

Significant $p$-values $(p<0.05)$ are indicated in bold. 
Table 2 Analyses Of Variance for Global Working Memory Capacity, Working Memory Subdomains, and Executive Working Memory Across all Investigated Polymorphisms

\begin{tabular}{|c|c|c|c|c|c|c|c|c|c|c|c|c|c|c|c|c|c|c|c|c|}
\hline & \multicolumn{5}{|c|}{ 5-HTTLPR } & \multicolumn{5}{|c|}{ VNTR $\ln 2$} & \multicolumn{5}{|c|}{ TPH2 (rs4570625) } & \multicolumn{5}{|c|}{ TPH2 (rsI386497) } \\
\hline & $\mathbf{F}$ & df & $P_{\text {ncor }}$ & $p^{2}$ & $P_{\text {cor }}$ & $\mathbf{F}$ & df & Pncor & $\eta \boldsymbol{P}^{2}$ & $P_{\text {cor }}$ & $\mathbf{F}$ & df & Pncor & $\eta p^{2}$ & $P_{\text {cor }}$ & $\mathbf{F}$ & df & Pncor & $\eta p^{2}$ & $P_{\text {cor }}$ \\
\hline \multicolumn{21}{|l|}{ Global WM capacity } \\
\hline Group & 11.96 & 1 & 0.001 & 0.054 & 0.002 & 26.48 & 1 & 0.000 & 0.114 & 0.000 & 23.59 & 1 & 0.000 & 0.103 & 0.000 & 10.78 & I & 0.001 & 0.049 & 0.001 \\
\hline Genotype & 0.47 & 2 & 0.626 & 0.004 & 0.999 & 0.52 & 2 & 0.593 & 0.005 & 0.999 & 0.13 & 1 & 0.724 & 0.001 & 0.999 & 0.07 & 1 & 0.791 & 0.000 & 0.791 \\
\hline Group $\times$ genotype & 4.87 & 2 & 0.009 & 0.044 & 0.018 & 6.54 & 2 & 0.002 & 0.060 & 0.006 & 2.30 & 1 & 0.131 & 0.011 & 0.131 & 6.24 & 1 & 0.013 & 0.029 & 0.026 \\
\hline \multicolumn{21}{|l|}{ Spatial WM (SWM) } \\
\hline Group & 5.39 & 1 & 0.021 & 0.025 & 0.042 & 13.52 & 1 & 0.000 & 0.062 & 0.001 & 14.29 & 1 & 0.000 & 0.065 & 0.000 & 3.33 & 1 & 0.069 & 0.016 & 0.069 \\
\hline Genotype & 2.99 & 2 & 0.053 & 0.028 & 0.212 & 0.70 & 2 & 0.498 & 0.007 & 0.999 & 0.02 & 1 & 0.899 & 0.000 & 0.899 & 0.33 & 1 & 0.566 & 0.002 & 0.999 \\
\hline Group $\times$ genotype & 3.18 & 2 & 0.044 & 0.029 & 0.088 & 4.02 & 2 & 0.019 & 0.038 & 0.057 & 3.23 & 1 & 0.074 & 0.016 & 0.074 & 11.15 & 1 & 0.001 & 0.050 & 0.004 \\
\hline \multicolumn{21}{|l|}{ Verbal WM (LNST) } \\
\hline Group & 7.93 & 1 & 0.005 & 0.036 & 0.005 & 17.55 & 1 & 0.000 & 0.079 & 0.000 & 16.04 & 1 & 0.000 & 0.072 & 0.000 & 8.17 & 1 & 0.005 & 0.037 & 0.009 \\
\hline Genotype & 0.88 & 2 & 0.417 & 0.008 & 0.999 & 0.91 & 2 & 0.403 & 0.009 & 0.999 & 0.23 & 1 & 0.635 & 0.001 & 0.999 & 0.03 & 1 & 0.859 & 0.000 & 0.859 \\
\hline Group $\times$ genotype & 3.26 & 2 & 0.040 & 0.030 & 0.120 & 3.75 & 2 & 0.025 & 0.035 & 0.100 & 1.72 & 1 & 0.191 & 0.008 & 0.191 & 4.08 & 1 & 0.045 & 0.019 & 0.090 \\
\hline \multicolumn{21}{|l|}{ Visuospatial WM (PAL) } \\
\hline Group & 6.68 & 1 & 0.010 & 0.031 & 0.010 & 12.85 & 1 & 0.000 & 0.056 & 0.001 & 9.68 & 1 & 0.002 & 0.045 & 0.006 & 7.88 & 1 & 0.005 & 0.036 & 0.011 \\
\hline Genotype & 0.32 & 2 & 0.727 & 0.003 & 0.999 & 0.28 & 2 & 0.760 & 0.003 & 0.999 & 0.10 & 1 & 0.755 & 0.000 & 0.999 & 0.03 & 1 & 0.854 & 0.000 & 0.854 \\
\hline Group $\times$ genotype & 2.19 & 2 & 0.115 & 0.020 & 0.345 & 3.22 & 2 & 0.042 & 0.030 & 0.168 & 0.01 & 1 & 0.909 & 0.000 & 0.909 & 0.06 & 1 & 0.803 & 0.000 & 0.999 \\
\hline \multicolumn{21}{|l|}{ Executive WM (Strategy) } \\
\hline Group & 0.63 & 1 & 0.428 & 0.003 & 0.428 & 5.81 & 1 & 0.017 & 0.028 & 0.068 & 5.39 & 1 & 0.021 & 0.026 & 0.063 & 1.84 & 1 & 0.177 & 0.009 & 0.354 \\
\hline Genotype & 0.86 & 2 & 0.427 & 0.008 & 0.999 & 3.07 & 2 & 0.048 & 0.029 & 0.192 & 0.09 & 1 & 0.766 & 0.000 & 0.999 & 0.00 & 1 & 0.964 & 0.000 & 0.964 \\
\hline Group $\times$ genotype & 5.55 & 2 & 0.004 & 0.050 & 0.016 & 4.88 & 2 & 0.009 & 0.045 & 0.027 & 0.66 & 1 & 0.417 & 0.003 & 0.417 & 1.84 & 1 & 0.176 & 0.009 & 0.352 \\
\hline
\end{tabular}

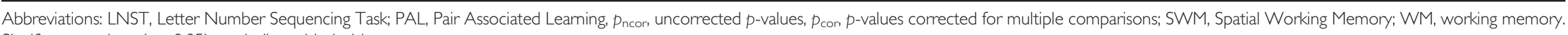
Significant $p$-values $(p<0.05)$ are indicated in bold. 


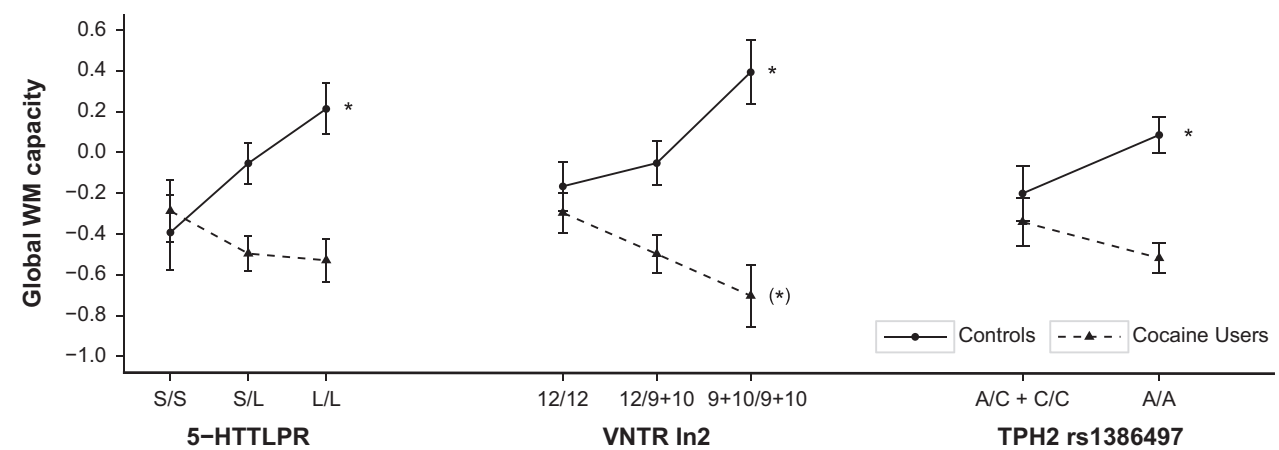

Figure I Global working memory capacity scores for the three polymorphisms with significant group $\times$ genotype interactions (mean and SE, z-transformed and corrected for age and years of education). Significant genotype effects $(p<0.05)$ within each group are indicated with *, whereas trends for genotype effects $(p<0.1)$ are indicated with $(*)$.

ADHD (all $p>0.05$ ). For additional information on the substance use of both groups, see Supplementary Table S1 in the Supplementary Material. Genotype frequencies of all four polymorphisms (see also Supplementary Table S2) were distributed in accordance with the Hardy-Weinberg Equilibrium and the SNPs rs4570625 and rs1386497 were found to be in minimal LD $\left(\mathrm{D}^{\prime}=1.0\right.$, LOD: $\left.5.16, r^{2}=0.06\right)$. None of the polymorphisms was associated with cocaine use per se (Supplementary Table S2) and within the group of cocaine users genotype groups did not differ regarding cocaine consumption (Supplementary Table S3).

\section{Global WM Capacity}

Global WM performance was affected by a significant main effect of the factor group, whereas the factor genotype alone was not significant in any analysis (Table 2). However, there were significant group $\times$ genotype interactions for all variants except for TPH2 rs4570625, which nonetheless showed a similar trend. Cocaine users revealed a worse WM performance compared with controls as shown previously for this sample (Vonmoos et al, 2013), whereby 5-HTTLPR, VNTR In2, and TPH2 rs1386497 genotype inversely affected WM in cocaine users and controls (Figure 1). These effects remained significant after correction for multiple comparisons (see Table 2) and even when age, years of education, BDI, and ADHD were introduced as covariates (5-HTTLPR: F $(2,207)=5.28, \quad p_{\text {cor }}<0.05 ; \quad$ VNTR In $: \quad \mathrm{F}(2,202)=7.29$, $p_{\text {cor }}<0.01 ; \quad$ TPH2 rs1386497: $\left.\mathrm{F}(1,207)=5.14, \quad p_{\text {cor }}<0.05\right)$. Among the covariates, age showed a significant main effect in the ANCOVAs of all three significant polymorphisms (all $p_{\text {cor }}<0.001$ ), whereas years of education was only significant for 5-HTTLPR and TPH2 rs1386497 (both $p_{\text {cor }}<0.05$ ).

\section{WM Subdomains and Executive WM}

Table 2 further reveals that the above described main effects of group and the group $\times$ genotype interactions were also found within the spatial, verbal, and visuospatial WM subdomains and in the executive WM parameter SMW strategy. In SWM strategy, we observed similar significant group $\times$ genotype interactions with 5-HTTLPR and VNTR In2 genotype as shown for global WM above (see Supplementary Figure S1). Regarding the spatial WM subdomain (CANTAB SWM total errors), significant interactions (corrected for multiple testing) were observed for VNTR $\ln 2$ and TPH2 rs1386497 genotypes,

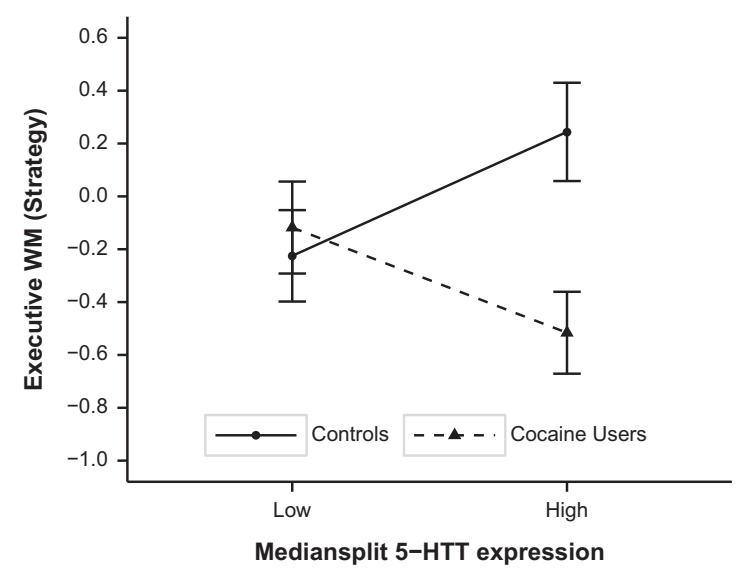

Figure 2 Executive working memory function (Spatial Working Memory strategy scores) in cocaine users and controls stratified for high vs low of 5-HTT mRNA expression (mean and SE, z-transformed and corrected for age and years of education).

whereas 5-HTTLPR and TPH2 rs4570625 displayed strong trends. LNST also showed moderate interaction effects of 5-HTTLPR, VNTR $\ln 2$ genotypes, and TPH2 rs1386497 with group, which remained trends after multiple-test correction. Finally, in CANTAB PAL, no interaction effects were strong enough to survive correction for multiple testing. It should be noted that all subdomains (with exception of PAL regarding TPH2 rs1386497) showed the same pattern of group $\times$ genotype interaction as illustrated for the global WM capacity index in Figure 1 (see Supplementary Figure S2).

\section{5-HTT mRNA Expression}

Peripheral mRNA expression did not differ between controls and cocaine users and across genotype groups (see Supplementary Table S4). Also, correlations between 5-HTT mRNA expression and WM performance did not reveal any significant results, neither in the total sample nor in both subgroups. However, using a median split of the mRNA expression (high $v s$ low expression) together with the factor group in a two-way ANOVA revealed a significant group $\times$ mRNA expression interaction $\left(\mathrm{F}(1,107)=6.36, p_{\text {cor }}>0.05\right.$, corrected for age and years of education) in SWM strategy (Figure 2). In addition, by including mRNA expression as a covariate in an ANCOVA together with group and genotype, we found significant three-way interactions of group $x$ 
genotype $\times$ mRNA expression for VNTR In 2 in the global WM capacity index $\left(\mathrm{F}(4,96)=5.04, p_{\text {cor }}<0.01\right)$, in LNST $\left(\mathrm{F}(4,96)=3.93, \quad p_{\mathrm{cor}}<0.05\right)$ and $\mathrm{PAL} \quad(\mathrm{F}(4,96)=3.70$, $\left.p_{\text {cor }}<0.05\right)$, for TPH2 rs4570625 in the global WM capacity index $\left(\mathrm{F}(2,101)=9.15, p_{\text {cor }}<0.001\right)$, in LNST $\left(\mathrm{F}(2,101)=4.85, \quad p_{\text {cor }}<0.05\right), \quad$ PAL $\quad(\mathrm{F}(2,101)=7.16$, $\left.p_{\text {cor }}<0.01\right)$, and SWM strategy $\left(\mathrm{F}(2,101)=5.51, p_{\text {cor }}<0.05\right)$ and for TPH2 rs1386497 in LNST $(\mathrm{F}(1,207)=4.401$, $\left.p_{\text {cor }}<0.05\right)$. These threefold interactions (see Supplementary Figures S3-S7) were mainly explained by a general interaction between gene expression and group mirroring the group $\times$ genotype interaction shown above (see Figure 2 ).

\section{DISCUSSION}

The present study was designed to investigate whether WM impairments in cocaine users are mediated by polymorphisms within the 5-HT system. We demonstrated that three of four investigated 5-HT-impacting polymorphisms (5-HTTLPR, VNTR In2, and TPH2 rs1386497) mediate WM performance and that their effects on WM depend on cocaine use of participants.

Concerning the 5-HTTLPR polymorphism, we found the long/long genotype to be a risk factor for the presence of WM impairments in cocaine users, although it was associated with improved WM performance in healthy controls. Even though the effects of 5-HTTLPR on 5-HT neurotransmission in humans is a subject of controversial debate, our results can be interpreted in consideration of previous animal studies. Carriers of the short allele of the 5-HTTLPR polymorphism are assumed to display decreased cerebral 5-HTT expression, and thus decreased 5-HT reuptake after synaptic signaling (Lesch et al, 1996; Glatz et al, 2003). However, carrying the short allele does not appear to result in increased 5-HT neurotransmission. In fact, increasing evidence from studies using 5-HTT knock-out mice suggests that having the short allele results in decreased 5-HT neurotransmission based on long-term developmental effects of reduced 5-HT reuptake on cell number, firing rate, and postsynaptic receptor sensitivity (Lira et al, 2003; Kim et al, 2005). Assuming, thus, that healthy control subjects carrying the short/short genotype have lower 5-HT activity and a reduced clearance rate after activation than long/long controls, our results are consistent with previous studies showing decreased cognitive functioning in participants with lower 5-HT levels (Murphy et al, 2002; Porter et al, 2003; Rogers et al, 2003; Allen et al, 2006; Fitzgerald, 2011). However, this interpretation is speculative because there is no consensus on the effects of 5-HTTLPR on 5-HT neurotransmission in humans so far. For instance, two molecular imaging studies employing PET in humans did not find an association between the 5-HTTLPR genotype and 5-HTT binding (Shioe et al, 2003; Murthy et al, 2010), whereas two other PET studies showed increased 5-HTT densities in putamen and midbrain, respectively, of long allele carriers (Praschak-Rieder et al, 2007; Reimold et al, 2007).

In cocaine users, carriers of the short/short genotype showed comparable WM performance as short/short genotype carriers of the controls. This finding is plausible considering the effects of cocaine use on the 5-HT system. Chronic cocaine administration is postulated (Müller and Homberg, 2015) to simultaneously result in an overexpression of 5-HTT at terminal sites of neurons and in an attenuated expression of 5-HTT at somatodendritic sites (Muller et al, 2002; Gould et al, 2011; Müller and Homberg, 2015). By this, chronic cocaine consumption is thought to result in a chronically reduced 5-HT neuronal activity together with a more efficient removal of 5-HT from the synaptic cleft in terminal areas of the 5-HT projections. The 5-HTTLPR long/long genotype probably displays more 5-HTT under basal conditions, which may offer more target sites for cocaine interactions. One may, therefore, speculate that 5-HTT adaptations after chronic cocaine use are stronger in the long/long than in the short/short genotype. This may result in a stronger reduction of basal 5-HT activity in the long/long genotype and, thus, in more pronounced WM deficits. It could, thus, be assumed that, under such conditions, having a genetically limited 5-HTT expression protects against cocaine-induced adaptations of the 5-HTT function. Hence, this view is supported by our data indicating unaffected WM performance in cocaine users carrying the short allele.

Our results regarding the 5-HTTLPR polymorphism seem to confirm the hypothesis that increased 5-HT activity is associated with improved cognitive functioning (in our case WM) in healthy controls (Murphy et al, 2002; Rogers et al, 2003). Moreover, we went one step further by showing that environmental factors (such as cocaine use) are able to moderate the direction of the polymorphism effects. This finding is especially interesting considering the conflicting results of previous studies suggesting that the effects of the 5-HTTLPR might be task specific (Anderson et al, 2012; Zilles et al, 2012). However, we were able to demonstrate consistent genotype effects across WM tasks measuring spatial (SWM), verbal (LNST), and visuospatial (PAL) WM subdomains (see Table 1 and Supplementary Figure S2). Hence, we propose that undetected gene $\times$ environment interactions (such as the discovered genotype-cocaine interaction) could provide an explanation for the previously conflicting results.

Our findings are further supported by the 5-HTT mRNA expression results. Analyzing peripheral mRNA expression in whole-blood samples, we found a similar group $\times$ genotype interaction effect of 5-HTTLPR in form of an interaction of group $\times$ mRNA expression also indicating higher WM performance in controls with increased 5-HTT expression and lower WM performance in cocaine users with increased 5-HTT expression. This is consistent with previous studies showing that 5-HTT mRNA expression can be used to replicate 5-HTTLPR genotype findings (Gardner et al, 2009).

Regarding the two SNPs in the TPH2 gene, we were not able to clearly replicate previous studies postulating the rare T/T genotype of rs4570625 to be associated with lower WM performance (Reuter et al, 2008). However, we found a significant interaction between TPH2 rs1386497 (which is in LD with rs4570625) and cocaine use. The A/A genotype of rs1386497 was associated with lower performance in cocaine users, whereas healthy controls carrying the A/A genotype showed higher WM performance. Tryptophan hydroxylase is a rate-limiting enzyme of the 5-HT synthesis (Kim et al, 2002) and although the rs 1386497 has not yet been reported in relation to WM, its A allele has been associated with ADHD and cognitive deficits in ADHD (Sheehan et al, 2005; Manor et al, 2008). Interestingly, we reported in our previous work that the co-occurrence of cocaine consumption and 
ADHD had a particularly negative impact on cognitive functioning (Vonmoos et al, 2013). However, including $\mathrm{ADHD}$ as covariate still revealed an ADHD-independent interaction effect of TPH2 rs1386497 and cocaine use on WM performance.

Concerning the VNTR In2, we demonstrated that controls revealed higher WM performance when carrying the short allele $(9+10 / 9+10$ copies) compared with carriers of the long allele (12/12 copies). In cocaine users, again, this pattern was inverted. It has been proposed that the VNTR In2 polymorphism acts as a transcriptional regulator of the 5-HTT expression but its exact effect is not fully clear yet (MacKenzie and Quinn, 1999). Comparing our findings of the 5-HTTLPR with the findings of the VNTR In2 reveals that the long/long genotype of 5-HTTLPR seems to have a similar effect on WM as the 12 copies variant of the VNTR In2. This specific pattern has already been found by other genetic studies with psychiatric patients suffering from personality and psychosomatic disorders (Kirtak et al, 2008; Garcia et al, 2010).

In sum, we presented evidence that genetic variations, putatively influencing 5-HT activity, mediate the development of WM impairments in cocaine users and that increased 5-HTT expression in the periphery goes along with these WM deficits. Our results support the view that chronic cocaine use enhances terminal 5-HTT function and that this enhanced activity, together with adaptations of postsynaptic 5-HT receptors, is responsible for the cognitive decline seen in cocaine users (Müller and Homberg, 2015). Alternatively, it might be speculated that specific 5-HTT polymorphisms, such as 5-HTTLPR and VNTR In2, impact the protein tertiary structure of the 5-HTT leading to a changed affinity to cocaine. In that case, genetic variations could also impact the acute effects of cocaine on 5-HT reuptake inhibition. Future studies should, therefore, investigate whether there are genotype differences in the acute effects of cocaine administration on a neuropharmacological as well as on a behavioral level. In addition, functional and molecular imaging techniques might be a promising approach to get a deeper understanding of the mechanisms underlying the cocaine-genotype interaction on WM.

In conclusion, we demonstrated that three 5-HT-impacting polymorphisms are risk alleles for the development of WM deficits in cocaine users. Hence, pharmacological compounds targeting 5-HT neurotransmission might be a promising treatment option of WM deficits in cocaine addiction. In addition, our results regarding the 5-HTTLPR and the VNTR In 2 polymorphisms in healthy controls seem to confirm the hypothesis (Fitzgerald, 2011) that increased 5-HT activity improves cognitive functioning (in our case WM). Finally, the discovered gene $\times$ environment interaction (ie, cocaine use moderated genotype effects) indicates that the complexity of the 5-HT systems requires us to discover other relevant factors to fully understand the involvement of 5-HT in WM processing.

\section{FUNDING AND DISCLOSURE}

The study was supported by grants from the Swiss National Science Foundation (SNSF; grant No. PP00P1-123516/1 and PP00P1-146326/1) and the Olga Mayenfisch Foundation. The authors declare no conflict of interest.

\section{ACKNOWLEDGMENTS}

We thank Vera Guttenthaler and Miryame Hofmann for technical assistance.

\section{REFERENCES}

Allen PP, Cleare AJ, Lee F, Fusar-Poli P, Tunstall N, Fu CH et al (2006). Effect of acute tryptophan depletion on pre-frontal engagement. Psychopharmacology (Berl) 187: 486-497.

American Psychiatric Association (1994). Diagnostic and Statistical Manual of Mental Disorders. 4th edn (DSM-IV) APA.

Anderson DE, Bell TA, Awh E (2012). Polymorphisms in the 5-HTTLPR gene mediate storage capacity of visual working memory. J Cogn Neurosci 24: 1069-1076.

Barrett JC, Fry B, Maller J, Daly MJ (2005). Haploview: analysis and visualization of $\mathrm{LD}$ and haplotype maps. Bioinformatics 21: 263-265.

Beveridge TJ, Gill KE, Hanlon CA, Porrino LJ (2008). Review. Parallel studies of cocaine-related neural and cognitive impairment in humans and monkeys. Philos Trans R Soc Lond B Biol Sci 363: 3257-3266.

Bosia M, Anselmetti S, Pirovano A, Ermoli E, Marino E, Bramanti P et al (2010). HTTLPR functional polymorphism in schizophrenia: executive functions vs. sustained attention dissociation. Prog Neuropsychopharmacol Biol Psychiatry 34: 81-85.

Dahl JP, Cubells JF, Ray R, Weller AE, Lohoff FW, Ferraro TN et al (2006). Analysis of variations in the tryptophan hydroxylase-2 (TPH2) gene in cocaine dependence. Addict Biol 11: 76-83.

Enge S, Fleischhauer M, Lesch KP, Reif A, Strobel A (2011). Serotonergic modulation in executive functioning: linking genetic variations to working memory performance. Neuropsychologia 49: 3776-3785.

Enoch MA, Gorodetsky E, Hodgkinson C, Roy A, Goldman D (2011). Functional genetic variants that increase synaptic serotonin and 5-HT3 receptor sensitivity predict alcohol and drug dependence. Mol Psychiatry 16: 1139-1146.

Fitzgerald PJ (2011). A neurochemical yin and yang: does serotonin activate and norepinephrine deactivate the prefrontal cortex? Psychopharmacology (Berl) 213: 171-182.

Garcia LF, Aluja A, Fibla J, Cuevas L, Garcia O (2010). Incremental effect for antisocial personality disorder genetic risk combining 5HTTLPR and 5-HTTVNTR polymorphisms. Psychiatry Res 177: 161-166.

Gardner KL, Hale MW, Lightman SL, Plotsky PM, Lowry CA (2009). Adverse early life experience and social stress during adulthood interact to increase serotonin transporter mRNA expression. Brain Res 1305: 47-63.

Glatz K, Mossner R, Heils A, Lesch KP (2003). Glucocorticoidregulated human serotonin transporter (5-HTT) expression is modulated by the 5-HTT gene-promotor-linked polymorphic region. J Neurochem 86: 1072-1078.

Goldstein RZ, Leskovjan AC, Hoff AL, Hitzemann R, Bashan F, Khalsa SS et al (2004). Severity of neuropsychological impairment in cocaine and alcohol addiction: association with metabolism in the prefrontal cortex. Neuropsychologia 42: 1447-1458.

Gould RW, Gage HD, Banks ML, Blaylock BL, Czoty PW, Nader MA (2011). Differential effects of cocaine and MDMA self-administration on cortical serotonin transporter availability in monkeys. Neuropharmacology 61: 245-251.

Grunblatt E, Bartl J, Zehetmayer S, Ringel TM, Bauer P, Riederer P et al (2009). Gene expression as peripheral biomarkers for sporadic Alzheimer's disease. J Alzheimers Dis 16: 627-634.

Hochberg Y (1988). A sharper Bonferroni procedure for multiple tests of significance. Biometrika 75: 3 .

Homberg JR, Lesch KP (2011). Looking on the bright side of serotonin transporter gene variation. Biol Psychiatry 69: 513-519. 
Hu XZ, Lipsky RH, Zhu G, Akhtar LA, Taubman J, Greenberg BD et al (2006). Serotonin transporter promoter gain-of-function genotypes are linked to obsessive-compulsive disorder. Am J Hum Genet 78: 815-826.

Hulka LM, Eisenegger C, Preller KH, Vonmoos M, Jenni D, Bendrick $\mathrm{K}$ et al (2014). Altered social and non-social decisionmaking in recreational and dependent cocaine users. Psychol Med 44: 1015-1028.

Jovanovski D, Erb S, Zakzanis KK (2005). Neurocognitive deficits in cocaine users: a quantitative review of the evidence. J Clin Exp Neuropsychol 27: 189-204.

Kim DK, Tolliver TJ, Huang SJ, Martin BJ, Andrews AM, Wichems C et al (2005). Altered serotonin synthesis, turnover and dynamic regulation in multiple brain regions of mice lacking the serotonin transporter. Neuropharmacology 49: 798-810.

Kim SW, Park SY, Hwang O (2002). Up-regulation of tryptophan hydroxylase expression and serotonin synthesis by sertraline. Mol Pharmacol 61: 778-785.

Kirtak N, Inaloz HS, Akcali C, Erdal E, Herken H, Yildirim M et al (2008). Association of serotonin transporter gene-linked polymorphic region and variable number of tandem repeat polymorphism of the serotonin transporter gene in lichen simplex chronicus patients with psychiatric status. Int J Dermatol 47: 1069-1072.

Lesch KP, Bengel D, Heils A, Sabol SZ, Greenberg BD, Petri S et al (1996). Association of anxiety-related traits with a polymorphism in the serotonin transporter gene regulatory region. Science 274: 1527-1531.

Lira A, Zhou M, Castanon N, Ansorge MS, Gordon JA, Francis JH et al (2003). Altered depression-related behaviors and functional changes in the dorsal raphe nucleus of serotonin transporterdeficient mice. Biol Psychiatry 54: 960-971.

MacKenzie A, Quinn J (1999). A serotonin transporter gene intron 2 polymorphic region, correlated with affective disorders, has allele-dependent differential enhancer-like properties in the mouse embryo. Proc Natl Acad Sci USA 96: 15251-15255.

Mannelli P, Patkar AA, Murray HW, Certa K, Peindl K, Mattila-Evenden $\mathrm{M}$ et al (2005). Polymorphism in the serotonin transporter gene and response to treatment in African American cocaine and alcohol-abusing individuals. Addict Biol 10: 261-268.

Manor I, Laiba E, Eisenberg J, Meidad S, Lerer E, Israel S et al (2008). Association between tryptophan hydroxylase 2, performance on a continuance performance test and response to methylphenidate in ADHD participants. Am J Med Genet B Neuropsychiatr Genet 147B: 1501-1508.

Matuskey D, Bhagwagar Z, Planeta B, Pittman B, Gallezot JD, Chen J et al (2013). Reductions in Brain 5-HT Receptor Availability in Primarily Cocaine-Dependent Humans. Biol Psychiatry 76: 816-822.

Muller CP, De Souza Silva MA, DePalma G, Tomaz C, Carey RJ, Huston JP (2002). The selective serotonin(1A)-receptor antagonist WAY 100635 blocks behavioral stimulating effects of cocaine but not ventral striatal dopamine increase. Behav Brain Res 134: 337-346.

Müller CP, Homberg JR (2015). The role of serotonin in drug use and addiction. Behav Brain Res 277: 146-192.

Murphy FC, Smith KA, Cowen PJ, Robbins TW, Sahakian BJ (2002). The effects of tryptophan depletion on cognitive and affective processing in healthy volunteers. Psychopharmacology (Berl) 163: 42-53.

Murthy NV, Selvaraj S, Cowen PJ, Bhagwagar Z, Riedel WJ, Peers P et al (2010). Serotonin transporter polymorphisms (SLC6A4 insertion/deletion and rs25531) do not affect the availability of 5-HTT to [11C] DASB binding in the living human brain. Neuroimage 52: 50-54.

Owen AM, Downes JJ, Sahakian BJ, Polkey CE, Robbins TW (1990). Planning and spatial working memory following frontal lobe lesions in man. Neuropsychologia 28: 1021-1034.
Patkar AA, Berrettini WH, Hoehe M, Hill KP, Gottheil E, Thornton CC et al (2002). No association between polymorphisms in the serotonin transporter gene and susceptibility to cocaine dependence among African-American individuals. Psychiatr Genet 12: 161-164.

Patkar AA, Berrettini WH, Hoehe M, Hill KP, Sterling RC, Gottheil E et al (2001). Serotonin transporter (5-HTT) gene polymorphisms and susceptibility to cocaine dependence among African-American individuals. Addict Biol 6: 337-345.

Porter RJ, Lunn BS, O'Brien JT (2003). Effects of acute tryptophan depletion on cognitive function in Alzheimer's disease and in the healthy elderly. Psychol Med 33: 41-49.

Praschak-Rieder N, Kennedy J, Wilson AA, Hussey D, Boovariwala A, Willeit $M$ et al (2007). Novel 5-HTTLPR allele associates with higher serotonin transporter binding in putamen: a [(11)C] DASB positron emission tomography study. Biol Psychiatry 62: 327-331.

Preller KH, Ingold $\mathrm{N}$, Hulka LM, Vonmoos $\mathrm{M}$, Jenni $\mathrm{D}$, Baumgartner MR et al (2013). Increased sensorimotor gating in recreational and dependent cocaine users is modulated by craving and attention-deficit/hyperactivity disorder symptoms. Biol Psychiatry 73: 225-234.

Puig MV, Gulledge AT (2011). Serotonin and prefrontal cortex function: neurons, networks, and circuits. Mol Neurobiol 44: 449-464.

Quednow BB, Kuhn KU, Hoenig K, Maier W, Wagner M (2004). Prepulse inhibition and habituation of acoustic startle response in male MDMA ('ecstasy') users, cannabis users, and healthy controls. Neuropsychopharmacology 29: 982-990.

Reimold M, Smolka MN, Schumann G, Zimmer A, Wrase J, Mann $\mathrm{K}$ et al (2007). Midbrain serotonin transporter binding potential measured with [11C]DASB is affected by serotonin transporter genotype. J Neural Transm 114: 635-639.

Reuter M, Esslinger C, Montag C, Lis S, Gallhofer B, Kirsch P (2008). A functional variant of the tryptophan hydroxylase 2 gene impacts working memory: a genetic imaging study. Biol Psychol 79: 111-117.

Robbins TW, Arnsten AF (2009). The neuropsychopharmacology of fronto-executive function: monoaminergic modulation. Annu Rev Neurosci 32: 267-287.

Rogers RD, Tunbridge EM, Bhagwagar Z, Drevets WC, Sahakian BJ, Carter CS (2003). Tryptophan depletion alters the decisionmaking of healthy volunteers through altered processing of reward cues. Neuropsychopharmacology 28: 153-162.

Sheehan K, Lowe N, Kirley A, Mullins C, Fitzgerald M, Gill M et al (2005). Tryptophan hydroxylase 2 (TPH2) gene variants associated with ADHD. Mol Psychiatry 10: 944-949.

Shioe K, Ichimiya T, Suhara T, Takano A, Sudo Y, Yasuno F et al (2003). No association between genotype of the promoter region of serotonin transporter gene and serotonin transporter binding in human brain measured by PET. Synapse 48: 184-188.

Strauss E, Sherman EM, Spreen O (2006). A CompendiumNeuropsychological Tests: Administration, Norms, and Commentary. 3rd edn, Oxford University Press.

Tristan-Noguero A, Fernandez-Castillo N, Roncero C, Sanchez-Mora C, Ramos-Quiroga JA, Daigre C et al (2013). Lack of association between the LPR and VNTR polymorphisms of the serotonin transporter gene and cocaine dependence in a Spanish sample. Psychiatry Res 210: 1287-1289.

Vandesompele J, De Preter K, Pattyn F, Poppe B, Van Roy N, De Paepe A et al (2002). Accurate normalization of real-time quantitative RT-PCR data by geometric averaging of multiple internal control genes. Genome Biol 3: RESEARCH0034.

Vonmoos M, Hulka LM, Preller KH, Jenni D, Baumgartner MR, Stohler R et al (2013). Cognitive dysfunctions in recreational and dependent cocaine users: role of attention-deficit hyperactivity disorder, craving and early age at onset. $\mathrm{Br} J$ Psychiatry 203: 35-43. 
Vonmoos M, Hulka LM, Preller KH, Minder F, Baumgartner MR, Quednow BB (2014). Cognitive impairment in cocaine users is drug-induced but partially reversible: evidence from a longitudinal study. Neuropsychopharmacology 39: $2200-2210$.

Vrana SL, Vrana KE, Koves TR, Smith JE, Dworkin SI (1993). Chronic cocaine administration increases CNS tyrosine hydroxylase enzyme activity and mRNA levels and tryptophan hydroxylase enzyme activity levels. J Neurochem 61: 2262-2268.

Wechsler DA (1997). Wechsler Memory Scale. 3rd edn, Manual Psychological Corporation.
Williams GV, Rao SG, Goldman-Rakic PS (2002). The physiological role of 5-HT2A receptors in working memory. J Neurosci 22: 2843-2854.

Wittchen HU, Wunderlich U, Gruschwitz S, Zaudig M (1997). SKID-I. Strukturiertes Klinisches Interview für DSM-IV Achse I: Psychische Störungen, Hogrefe, Göttingen.

Zilles D, Meyer J, Schneider-Axmann T, Ekawardhani S, Gruber E, Falkai P et al (2012). Genetic polymorphisms of 5-HTT and DAT but not COMT differentially affect verbal and visuospatial working memory functioning. Eur Arch Psychiatry Clin Neurosci 262: $667-676$.

Supplementary Information accompanies the paper on the Neuropsychopharmacology website (http://www.nature.com/npp) 